STRUCTURAL BIOLOGY COMMUNICATIONS

ISSN 2053-230X

\section{Macromolecular crystallography and what it can contribute to antiparasite drug discovery}

\author{
W. N. Hunter ${ }^{\mathrm{a} *}$ and Manfred S. Weiss ${ }^{\mathrm{b}}$ \\ aDivision of Biological Chemistry and Drug Discovery, College of Life Sciences, University of Dundee, Dundee \\ DD1 5EH, Scotland, and ${ }^{\mathbf{b}}$ Helmholtz-Zentrum Berlin für Materialien und Energie, Macromolecular \\ Crystallography (HZB-MX), Albert-Einstein-Strasse 15, D-12489 Berlin, Germany. *Correspondence e-mail: \\ w.n.hunter@dundee.ac.uk
}

This issue of Acta Crystallographica Section F is dedicated towards structural investigations on proteins associated with molecular parasitology, specifically research linked to protozoan pathogens. It is in line with the previous special sections or issues contributed by the RIKEN-UK structural genomics consortium (December 2009), the Joint Center for Structural Genomics in October 2010 (Weiss \& Einspahr, 2010) and the Seattle Structural Genomics Center for Infectious Diseases in September 2011 (Weiss \& Einspahr, 2011). In contrast to the previous issues however, it is thematically much more focused on protozoan pathogens. Interest in these primitive eukaryotic organisms is often to exploit them as model systems and many significant advances have resulted from such research. As examples there are the fundamentally important discoveries relating to RNA editing, antigenic variation and the occurrence of glycosylphosphatidylinositol tags that anchor proteins on the cell surface, all accrued from research with the African trypanosome. However, a major impetus to study protists is the drastic toll they take on human life. The World Health Organization provide documentation and sobering statistics on the diseases and their impact on human health. For example http:// www.who.int/mediacentre/factsheets/fs375/en/ is their recently updated information sheet on Leishmaniasis. Researchers have sought to understand the processes that allow pathogens to exist, to invade a host, to evade the immune response, and to cause debilitating and often fatal diseases. Extending from such investigations and alongside drug discovery efforts enter structural biology, in particular crystallography and the high quality chemical information it can provide.

Remember that most drugs are smallish organic molecules that exert their beneficial effect by interacting with certain macromolecules. The discovery of such molecules has transformed human existence. Indeed the emergence of the modern pharmaceutical industry, founded as it was on the basis of antimicrobial and analgesic agents, might be considered one of the major developments of the last 100 years. Different disciplines contribute to drug discovery, and increasingly the search is on for new targets, better technologies and model systems to assist the process. Structural studies contribute a key role by providing accurate chemical information to guide assessment of targets, the development of reliable structure-activity relationships or templates for computational design projects in the search for lead compounds. There are numerous excellent studies published in which specific parasite systems have been targeted and dissected. In addition structural genomics efforts have involved cohorts of protist proteins and the Protein Data Bank has been greatly enriched in this area with both NMR and crystal structures.

We now present an issue of fundamental studies in molecular parasitology and also research directed toward protozoan drug target characterization. To set the scene there is an overview and perspective from Professor Wim Hol. In this he also details a few of his favourite examples where structural information contributes to the search for improved therapies. We commissioned Wim for an overview because of his outstanding contributions and infectious enthusiasm for the area; he continues to be an inspirational champion for the role of crystallography in drug discovery. There is also a short review from Professor Inari Kursula on cytoskeletal proteins related to the infection process. We thought it relevant to address this aspect and would highlight this as one in which there may be opportunities in future drug discovery work. We are pleased to also have a 
number of communications from structural genomics efforts which sit comfortably alongside more focused projects.

Section F's mandate is to allow the structural biology community to efficiently publish their data and we hope this special issue will serve to inform our community and perhaps others in the life sciences or medicinal chemistry areas, generate interest and provide encouragement to consider the journal as a natural home for results in molecular parasitology.
We look forward to seeing more structural information on parasite proteins, more advances in basic biology and results to support future efforts in early stage drug discovery.

\section{References}

Weiss, M. S. \& Einspahr, H. (2010). Acta Cryst. F66, 1406. Weiss, M. S. \& Einspahr, H. (2011). Acta Cryst. F67, 1159. 